12 Seiffert M, Schulz A, Ohl S, Dohner H, Stilgenbauer S, Lichter P. Soluble CD14 is a novel monocyte-derived survival factor for chronic lymphocytic leukemia cells, which is induced by CLL cells in vitro and present at abnormally high levels in vivo. Blood 2010; 116: 4223-4230.

13 Ziegler-Heitbrock L, Ancuta P, Crowe S, Dalod M, Grau V, Hart DN et al. Nomenclature of monocytes and dendritic cells in blood. Blood 2010; 116: e74-e80.

14 van RN, Hendrikx E. Liposomes for specific depletion of macrophages from organs and tissues. Methods Mol Biol 2010; 605: 189-203.

15 Hanna BS, McClanahan F, Yazdanparast H, Zaborsky N, Kalter V Rößner P et al. Depletion of CLL-associated patrolling monocytes and macrophages controls disease development and repairs immune dysfunction in vivo. Leukemia 2016; 30: 570-579.

c) (1) $\Theta$ This work is licensed under a Creative Commons AttributionNC ND NonCommercial-NoDerivs 4.0 International License. The images or other third party material in this article are included in the article's Creative Commons license, unless indicated otherwise in the credit line; if the material is not included under the Creative Commons license, users will need to obtain permission from the license holder to reproduce the material. To view a copy of this license, visit http:// creativecommons.org/licenses/by-nc-nd/4.0/

Supplementary Information accompanies this paper on the Leukemia website (http://www.nature.com/leu)

\title{
OPEN
}

\section{Efficacy of ruxolitinib on hepatomegaly in patients with myelofibrosis}

Leukemia (2016) 30, 1413-1415; doi:10.1038/leu.2015.310

The Janus kinase $1 / 2$ inhibitor ruxolitinib has been shown in a pivotal placebo-controlled phase III study (Controlled Myelofibrosis Study with Oral JAK Inhibitor Treatment I (COMFORT-I)) to significantly reduce splenomegaly and symptoms in patients with intermediate-2 or high-risk myelofibrosis (MF). Although splenomegaly is the most common manifestation of MF-associated extramedullary hematopoiesis, up to $65 \%$ of patients may also have hepatomegaly, ${ }^{1,2}$ which is often highly symptomatic and can lead to serious complications. ${ }^{3}$ Here, we present the results from a COMFORT-I post hoc analysis that assessed changes in liver volume and their relationship with concomitant changes in splenomegaly and measures of metabolic status.

As previously reported, ${ }^{4}$ COMFORT-I was a randomized, double-blind, placebo-controlled phase III study in patients with intermediate-2 or high-risk MF and a baseline spleen length $\geqslant 5 \mathrm{~cm}$ below the left costal margin. Patients randomized to placebo were allowed to cross over to ruxolitinib if they met prespecified criteria for disease progression. The primary end point was the proportion of patients who achieved $a \geqslant 35 \%$ reduction in spleen volume at week $24 .{ }^{4}$ Liver and spleen volumes were determined by magnetic resonance imaging or computed tomography per protocol; palpable liver length was not assessed. Percentage changes of spleen and liver volumes from baseline to week 144 were calculated for observed cases. For patients who crossed over from placebo to ruxolitinib, liver volume values obtained after crossover were excluded from the analysis for placebo and analyzed separately. In these individuals, baseline was defined as the last observation before crossover. Percentage changes in liver volume at week 24 were compared between treatment arms by analysis of covariance, with baseline liver volume as the covariate. Correlations of clinical parameters were assessed by Pearson's correlation coefficient with associated test for zero correlation and the $R^{2}$ statistic calculated by simple linear regression. A $5 \%$ significance level was applied to all statistical tests, without adjustment for multiple testing.

A total of 139 of 155 patients randomized to ruxolitinib and 105 of 154 patients randomized to placebo had evaluable liver volume data at baseline and week 24. Median (range) liver volume at baseline was $2452 \mathrm{~cm}^{3}\left(1268-4833 \mathrm{~cm}^{3}\right)$ in the ruxolitinib arm, and $2485 \mathrm{~cm}^{3}\left(1298-5230 \mathrm{~cm}^{3}\right)$ in the placebo arm (see Supplementary Table S1 for additional baseline characteristics). At week 24, patients treated with ruxolitinib had a mean percentage change (decrease) in liver volume of $-7.9 \%$ (median change, $-8.7 \%$; range, $-26.0 \%$ to $+17.5 \%$ ) compared with a mean increase of $3.5 \%$ (median change, $4.1 \%$; range, $36.0 \%$ to $+41.7 \%$ ) in the placebo arm $(P<0.0001$; Figures $1 \mathrm{a}$ and $\mathrm{b})$. The extent of liver volume reductions tended to be greater among patients who achieved average doses of 20 or $25 \mathrm{mg}$ twice daily during weeks 21-24 (mean changes at week $24:-9.7 \%$ and $-9.8 \%$, respectively) than among patients with lower average doses during weeks 21-24 (mean changes: $-5.1 \%$ with $<10 \mathrm{mg}$ twice daily, $-6.0 \%$ with $10 \mathrm{mg}$ twice daily and $-6.5 \%$ with $15 \mathrm{mg}$ twice daily).

In the ruxolitinib arm, liver volumes decreased rapidly during the first 12 weeks and continued to decrease until approximately week 48 , with a mean percentage change of $-10.6 \%$ at week 48 (range, -35.9 to +23.0 ). Improvement was maintained through week 144 (Figure 1c). In contrast, in the placebo group, liver volume increased over time, resulting in a mean percentage change of $6.0 \%$ at week 48 (range, $-21.6 \%$ to $+43.0 \%$ ) when most patients in the placebo group had discontinued or crossed over to ruxolitinib. Patients who crossed over from placebo to ruxolitinib experienced decreases in liver volume from the point of crossover, with an overall time course similar to that observed in patients randomized to ruxolitinib (Figure 1c).

We sought to determine whether the percentage changes in liver volume in the ruxolitinib arm were affected by baseline liver volume. Patients in the first $(n=37)$, second $(n=35)$, third $(n=36)$ and fourth $(n=31)$ quartile had median (range) baseline liver volumes of $1929 \mathrm{~cm}^{3}\left(1268-2115 \mathrm{~cm}^{3}\right), 2323 \mathrm{~cm}^{3}$ $\left(2131-2483 \mathrm{~cm}^{3}\right), \quad 2661 \mathrm{~cm}^{3}\left(2488-3014 \mathrm{~cm}^{3}\right)$ and $3417 \mathrm{~cm}^{3}$ $\left(3028-4833 \mathrm{~cm}^{3}\right)$, respectively. Patients experienced similar reductions in hepatomegaly at week 24 regardless of baseline liver volume, with mean \pm s.d. percentage changes in liver volume of $-8.3 \% \pm 7.9 \%,-6.4 \% \pm 7.5 \%,-7.8 \% \pm 8.6 \%$ and $-9.1 \% \pm 8.8 \%$ in the first, second, third and fourth quartile, respectively. Changes in liver volume over time observed for the entire study population were reflected in each quartile (Figure 1d). Although the mean values for each quartile showed some differences after week 60, the interpatient variability in each quartile (represented by the 
a

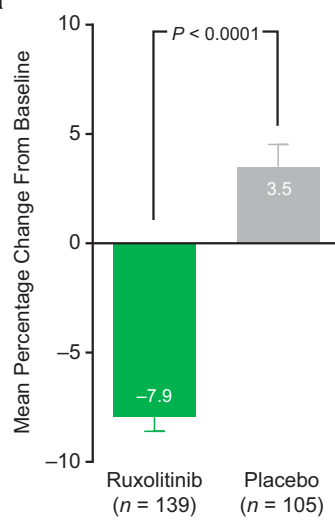

b

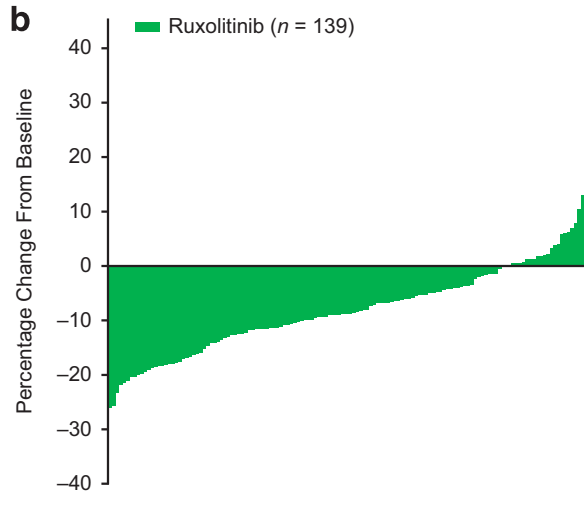

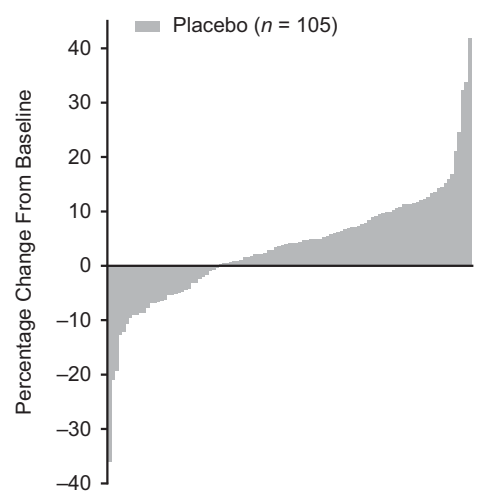

C

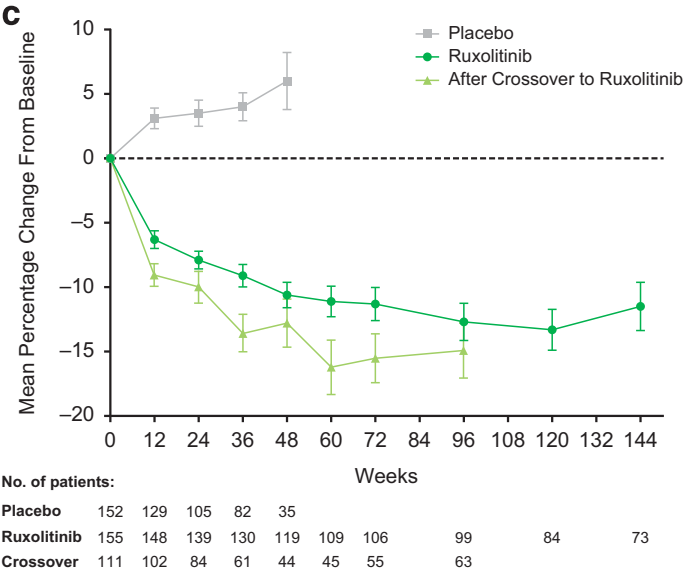

d

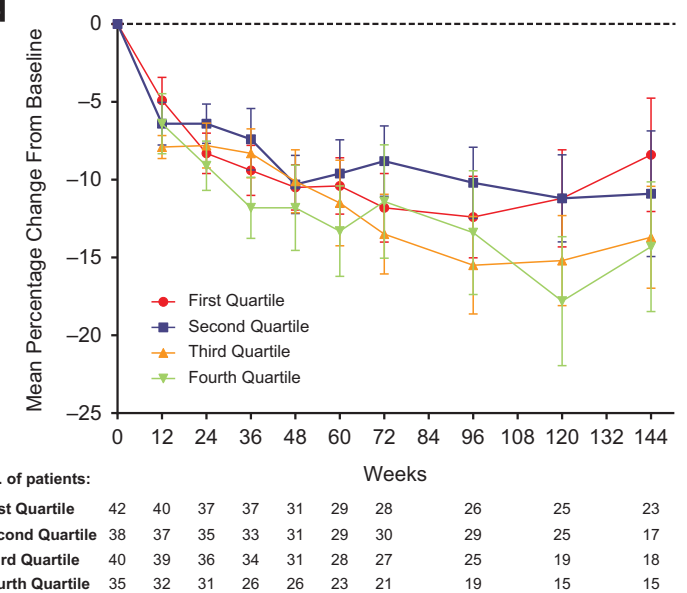

Figure 1. Changes in liver volume in COMFORT-I: (a) overall mean percentage change at week 24 by randomized treatment; (b) the percentage changes in individual patients at week 24 by randomized treatment; (c) the mean percentage changes over time by randomized treatment and in patients who crossed over from placebo to ruxolitinib; (d) the mean percentage changes over time by baseline liver volume quartile in the ruxolitinib arm.

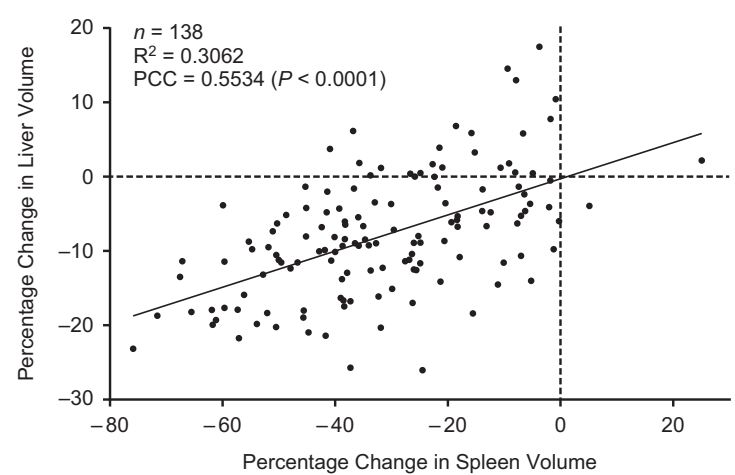

Figure 2. Correlation between percentage changes from baseline to week 24 in liver and spleen volume in patients randomized to ruxolitinib. PCC, Pearson's correlation coefficient.

standard error) was greater than the interquartile variability of the means.

Percentage changes in liver volume at week 24 significantly correlated with changes in splenomegaly, both for the entire evaluable population $\left(n=138 ; R^{2}=0.3062\right.$; Pearson correlation coefficient $(P C C)=0.5534 ; P<0.0001$; Figure 2 ) and for patients with both baseline liver and spleen volumes in the third or fourth quartile $\left(n=44 ; R^{2}=0.2284 ; \quad P C C=0.4779 ; \quad P=0.0010\right)$.
Nearly all patients who experienced a liver volume reduction also had a spleen volume reduction (Figure 2). Significant negative correlations were observed between changes in liver volume at week 24 and corresponding changes in body weight $\left(R^{2}=0.0446\right.$; $\mathrm{PCC}=-0.2111 ; \quad P=0.0129)$, leptin $\left(R^{2}=0.1036 ; \quad P C C=-0.3219\right.$; $P=0.0001)$ and albumin $\left(R^{2}=0.0472 ; P C C=-0.2172 ; P=0.0102\right)$. Increases in these parameters were observed in most patients who had a decrease in liver volume (Supplementary Figure S1).

The results of this exploratory analysis from COMFORT-I, a placebo-controlled study with a large study population, provide evidence suggesting that ruxolitinib is effective in providing rapid and sustained reduction in MF-related hepatomegaly. This has not been objectively demonstrated to date with other medical therapies. Overall, patients achieved most of their treatment benefit regarding the magnitude of liver volume reductions after $\sim 48$ weeks of treatment. By comparison, the time frame for maximal mean spleen volume responses in the COMFORT studies was $\sim 24$ weeks. ${ }^{4,5}$ Percentage reductions in liver volume were largely independent of the liver volume at baseline. Improvement in hepatomegaly was associated with previously reported improvements in splenomegaly, weight gain and increases in albumin and leptin, ${ }^{4,6}$ raising the possibility of shared underlying mechanisms. The correlation between liver volume reductions and increases in albumin levels is notable, as albumin is a measure of the biosynthetic function of the liver.

Reduction of hepatomegaly would be a benefit of particular clinical significance for patients undergoing splenectomy, as most of 
these patients eventually develop symptomatic hepatomegaly. ${ }^{7}$ As COMFORT-I was not designed to evaluate liver volume reductions in patients who had undergone splenectomy, we cannot speculate about the efficacy of ruxolitinib in alleviating hepatomegaly in these patients. However, maximum liver size reductions of $50-68 \%$ (based on palpation) have been observed in three patients with symptomatic hepatomegaly (palpable length, $14-16 \mathrm{~cm}$ ) and previous splenectomy who were treated with ruxolitinib in the phase II study. ${ }^{8}$

Our study used volumetric liver size assessment based on magnetic resonance imaging and computed tomography scans to evaluate the treatment effect of ruxolitinib on liver size compared with placebo. A considerable advantage of this approach over palpation is that it allows for the greatest precision and accuracy in the quantitative measurement of liver size. However, because of substantial interindividual variability in healthy individuals, liver volume alone is not always a sufficient indicator of the degree of hepatomegaly. A recent study estimated the mean volume \pm s.d. of enlarged livers at $2.32 \pm 0.75$ I compared with $1.51 \pm 0.25$ I for normal livers. ${ }^{9}$ This suggests that a majority of patients in COMFORT-I, including most, if not all, in the second, third and fourth baseline liver volume quartiles of the ruxolitinib arm had mild to massive hepatomegaly at baseline. Although liver volume reductions were observed across quartiles, the clinical significance of a $6-9 \%$ reduction in liver volume is limited by the lack of a corresponding patient-reported outcome measure to assess reduction in hepatomegaly-related symptoms. However, the correlations between reductions in spleen size and liver volume observed in this analysis along with previously published results showing a relationship between reductions in spleen volume and MF-related symptoms, ${ }^{10}$ and the correlations between liver volume and albumin and weight suggest that the reductions in hepatomegaly are clinically meaningful.

In conclusion, ruxolitinib provided significant reductions in liver volume compared with placebo in COMFORT-I. Liver volumes decreased rapidly during the first 12 weeks and continued to decrease through week 60, with benefit maintained with longerterm treatment. Percentage reductions in liver volume were associated with improvements in splenomegaly and metabolic status. Overall, these findings suggest that ruxolitinib may improve MF-related hepatomegaly.

\section{CONFLICT OF INTEREST}

SV, EA, JM, VG, RM and JG participated in an advisory board for Incyte Corporation and their Institutions received research funding from Incyte Corporation for conduct of COMFORT-I. HS and MM are employees of and have stock in Incyte Corporation. VG served on the advisory board of Novartis and received lecture fees from Incyte Corporation and Novartis.

\section{ACKNOWLEDGEMENTS}

The MD Anderson Cancer Center receives a support grant from the National Cancer Institute of the National Institutes of Health (P30 CA016672). JG is supported by the Charles and Ann Johnson Foundation. Medical writing assistance for this manuscript was provided by Roland Tacke, PhD, of Evidence Scientific Solutions and funded by Incyte Corporation.

\section{AUTHOR CONTRIBUTIONS}

SV, EA, JM, MM, VG, RM, and JG contributed to the study design and data collection, and HS conducted the data analyses. All authors contributed to the data interpretation and preparation of the manuscript and approved the final version of the manuscript.

\section{S Verstovsek ${ }^{1}$, E Atallah ${ }^{2}$, J Mascarenhas ${ }^{3}, \mathrm{H} \mathrm{Sun}^{4}, \mathrm{M} \mathrm{Montgomery}^{4}$, V Gupta ${ }^{5}$, R Mesa $^{6}$ and J Gotlib ${ }^{7}$ \\ ${ }^{1}$ Department of Leukemia, MD Anderson Cancer Center, Houston, TX, USA; \\ ${ }^{2}$ Department of Internal Medicine, Medical College of Wisconsin, Milwaukee, WI, USA; \\ ${ }^{3}$ Tisch Cancer Institute, Icahn School of Medicine at Mount Sinai, New York, NY, USA; \\ ${ }^{4}$ Incyte Corporation, Wilmington, DE, USA; \\ ${ }^{5}$ Department of Medicine, Princess Margaret Cancer Centre, Toronto, Ontario, Canada; \\ ${ }^{6}$ Division of Hematology \& Medical Oncology, Mayo Clinic Cancer Center, Scottsdale, AZ, USA and \\ ${ }^{7}$ Department of Medicine/Division of Hematology, Stanford University School of Medicine/Stanford Cancer Institute, \\ Stanford, CA, USA \\ E-mail: sverstov@mdanderson.org}

\section{REFERENCES}

1 Cervantes F, Dupriez B, Pereira A, Passamonti F, Reilly JT, Morra E et al. New prognostic scoring system for primary myelofibrosis based on a study of the International Working Group for Myelofibrosis Research and Treatment. Blood 2009; 113: 2895-2901.

2 Passamonti F, Cervantes F, Vannucchi AM, Morra E, Rumi E, Pereira A et al. A dynamic prognostic model to predict survival in primary myelofibrosis: a study by the IWG-MRT (International Working Group for Myeloproliferative Neoplasms Research and Treatment). Blood 2010; 115: 1703-1708.

3 Mughal TI, Vaddi K, Sarlis NJ, Verstovsek S. Myelofibrosis-associated complications: pathogenesis, clinical manifestations, and effects on outcomes. Int J Gen Med 2014; 7: 89-101.

4 Verstovsek S, Mesa RA, Gotlib J, Levy RS, Gupta V, DiPersio JF et al. A double-blind, placebo-controlled trial of ruxolitinib for myelofibrosis. N Engl J Med 2012; 366: 799-807.

5 Harrison C, Kiladjian JJ, Al-Ali HK, Gisslinger H, Waltzman R, Stalbovskaya V et al. JAK inhibition with ruxolitinib versus best available therapy for myelofibrosis. N Engl J Med 2012; 366: 787-798.

6 Mesa RA, Verstovsek S, Gupta V, Mascarenhas JO, Atallah E, Burn T et al. Effects of ruxolitinib treatment on metabolic and nutritional parameters in patients with myelofibrosis from COMFORT-I. Clin Lymphoma Myeloma Leuk 2015; 15: 214-221.e1

7 Mesa RA, Nagorney DS, Schwager S, Allred J, Tefferi A. Palliative goals, patient selection, and perioperative platelet management: outcomes and lessons from 3 decades of splenectomy for myelofibrosis with myeloid metaplasia at the Mayo Clinic. Cancer 2006; 107: 361-370.

8 Benjamini O, Jain P, Estrov Z, Kantarjian HM, Verstovsek S. Therapeutic effects of ruxolitinib in patients with myelofibrosis without clinically significant splenomegaly. Blood 2012; 120: 2768-2769.

9 Linguraru MG, Sandberg JK, Jones EC, Petrick N, Summers RM. Assessing hepatomegaly: automated volumetric analysis of the liver. Acad Radiol 2012; 19: 588-598.

10 Mesa RA, Shields A, Hare T, Erickson-Viitanen S, Sun W, Sarlis NJ et al. Progressive burden of myelofibrosis in untreated patients: assessment of patient-reported outcomes in patients randomized to placebo in the COMFORT-I study. Leuk Res 2013; 37: 911-916.

Supplementary Information accompanies this paper on the Leukemia website (http://www.nature.com/leu) 